February, 1993

LBL-33833

hep-ph/9303282

\title{
First Order Phase Transition in a Two Higgs-doublet Model with $M_{h}>M_{W}$
}

\author{
Vidyut Jain and Aris Papadopoulos \\ Theoretical Physics Group, \\ Lawrence Berkeley Laboratory, \\ 1 Cyclotron Road, Berkeley, California 94720
}

\begin{abstract}
We study the 3d effective field theory of a weakly coupled two Higgs-doublet scalar model at high temperature. Our model has three scalar quartic couplings and an $O(4)$ symmetry which is spontaneously broken by a nonzero scalar field vev at zero temperature. Using high temperature perturbation theory, renormalization group arguments in $4-\epsilon$ dimensions, as well as $1 / N$ expansion techniques in 3 dimensions, we argue that the transition from the high temperature symmetry restoring phase to the low temperature phase is first order for a range of scalar couplings. This result is not due to gauge couplings. We discuss the implications of our results for the transition in the two Higgs-doublet electroweak model, especially when the lightest neutral Higgs is heavier than the W-bosons.
\end{abstract}

${ }^{*}$ This work was supported by the Director, Office of Energy Research, Office of High Energy and Nuclear Physics, Division of High Energy Physics of the U.S. Department of Energy under contract DEAC03-76SF00098 and in part by the National Science Foundation under grant PHY-90-21139. 


\section{Disclaimer}

This document was prepared as an account of work sponsored by the United States Government. Neither the United States Government nor any agency thereof, nor The Regents of the University of California, nor any of their employees, makes any warranty, express or implied, or assumes any legal liability or responsibility for the accuracy, completeness, or usefulness of any information, apparatus, product, or process disclosed, or represents that its use would not infringe privately owned rights. Reference herein to any specific commercial products process, or service by its trade name, trademark, manufacturer, or otherwise, does not necessarily constitute or imply its endorsement, recommendation,or favoring by the United States Government or any agency thereof, or The Regents of the University of California. The views and opinions of authors expressed herein do not necessarily state or reflect those of the United States Government or any agency thereof of The Regents of the University of California and shall not be used for advertising or product endorsement purposes.

Lawrence Berkeley Laboratory is an equal opportunity employer. 
Introduction. The topic of high temperature phase transitions in $4 \mathrm{~d}$ scalar models has attracted a lot of interest recently due to the possibility of baryogenesis during the weak phase transition. It has been argued that baryogenesis requires a sufficiently strong first order phase transition $[1,2,3,4]$.

The nature of the phase transition can be determined if one has a reliable determination of the finite temperature effective scalar potential $V_{e f f}$. It has been known for some time $[7,8,9]$ that at sufficiently high temperature the ground state of a $\phi^{4}$ model is symmetry preserving with $\langle\phi\rangle=0$, even if the zero temperature ground state is not. What is not well understood is how the transition from the high temperature phase to the low temperature phase proceeds in generic models with several couplings.

One can imagine two possibilities as the temperature is lowered from a very high value: i) the minimum remains at $\langle\phi\rangle=0$ until a temperature $T_{1}$ at which point it jumps discontinuously to nonzero values $|<\phi>| \neq 0$, or ii) the minimum remains at $<$ $\phi>=0$ until a temperature $T_{2}$ at which it moves continuously away from the origin as the temperature is lowered further. Case i) indicates a first order transition and case ii) indicates a second order transition.

The determination of the transition order is not always easy due to infrared divergences in high $T$ field theory which can render perturbation theory useless in calculating $V_{\text {eff }}$ too close to the origin and at temperatures near the transition temperature. In this case, one can i) try resuming perturbation theory or ii) use renormalization group (RG) methods or iii) use $1 / N$ expansion techniques to gain some insight. When a model has several couplings, any one method cannot be trusted to give a reliable description of the transition for all values of the (perturbative) couplings.

The most thoroughly investigated electroweak model, the minimal standard model, can be argued [10-14] to give a first order transition for $M_{\text {Higgs }}$ sufficiently smaller than $M_{W}$. In this case, high $T$ perturbation theory can give a good description of the transition if used carefully.

The leading expression for the high temperature effective potential when $M_{\text {Higgs }} \ll M_{W}$

\footnotetext{
${ }^{\dagger}$ What we mean by $V_{\text {eff }}$ is the sum of one-particle irreducible diagrams with no external legs. This quantity need be neither real nor convex [5], in contrast to the standard definition of the effective potential given in many textbooks [6]. However, the minimum of the real part of our effective potential still gives the ground state of the theory.
} 
is of the form $[7,15,10-14]$

$$
V_{e f f}=m^{2}(T)|\Phi|^{2}-\delta T\left|\Phi^{3}\right|+\frac{\lambda(T)}{4 !}|\Phi|^{4},
$$

where $\Phi$ is the Higgs doublet, $\delta$ is proportional to the cube of the $S U(2)$ gauge coupling and $m^{2}(T) \propto\left(T^{2}-T_{2}^{2}\right)$. This potential describes a first order transition whose minimum jumps discontinuously to a value $\propto \delta T / \lambda$ at a temperature $T_{1}>T_{2}$. RG methods combined with $\epsilon$-expansion techniques $[15,16]$ as well as computations of $V_{\text {eff }}$ using $1 / N$ techniques (when $M_{W} \sim N M_{\text {Higgs }}$ ) $[17,18]$ are consistent with this result.

With the current experimental bounds on $M_{\text {Higgs }}$ [19], the situation $M_{\text {Higgs }} \geq O\left(M_{W}\right)$ is the one of physical interest. Unfortunately, for $M_{\text {Higgs }} \geq M_{W}$, perturbation theory breaks down and there is really no clear picture of what happens. However, one expects that at best the phase transition will be very weakly first order.

It has been suggested [11] that one should augment the minimal standard model with extra scalars in order to produce a big enough first order effect for values of the Higgs mass larger than the current experimental bound. The idea is to enlarge the ratio $\delta / \lambda$ during the phase transition.

One suggestion [11], is to add an electroweak singlet scalar $S$ with a large enough coupling to the Higgs doublet $\Phi$ of the form $\xi \Phi^{\dagger} \Phi S^{2}$. It has been argued that if $S$ is massive enough it contributes to $V_{\text {eff }}(T=0)$ in a way that reduces the value of $\lambda$ without decreasing $M_{\text {Higgs }}$.

Another nonminimal model is the two Higgs-doublet electroweak model. Due to the number of unknown parameters in this model it makes sense to study a toy model before attempting to understand the nature of the transition in the general case. In this letter we will study such a toy model. In particular, we study the $4 \mathrm{~d}$ weakly coupled scalar model with tree potential

$$
V\left(\phi_{1}, \phi_{2}\right)=\frac{\lambda}{4 !}\left[\left(\phi_{1}^{2}-v^{2}\right)^{2}+\left(\phi_{2}^{2}-v^{2}\right)^{2}\right]+\frac{\xi}{12} \phi_{1}^{2} \phi_{2}^{2}+\frac{\alpha}{6}\left(\phi_{1} \cdot \phi_{2}\right)^{2},
$$

where $\lambda>\xi$ (to ensure real physical masses) and $\phi_{1}$ and $\phi_{2}$ are both $O(4)$ vectors. Unless otherwise stated, we assume all couplings are arbitrarily small (their ratios, however, may not be) and nonnegative.

The model (2) has an $O(4)$ symmetry as well as the discrete symmetry $\phi_{1} \leftrightarrow \phi_{2}$. It is also invariant under $\phi_{1} \rightarrow-\phi_{1}$ or $\phi_{2} \rightarrow-\phi_{2}$. The potential (2) is a subset of the 
general potential describing the two Higgs-doublet model which has five quartic couplings [20], not three. One of the additional couplings breaks the $Z_{2}$ symmetry $\phi_{1} \leftrightarrow \phi_{2}$, the other is allowed because the potential needs to be invariant only under $S U(2) \times U(1)$, not $O(4) \sim S U(2) \times S U(2)$.

Although the 2 Higgs-doublet model has been studied at one-loop by previous authors [21], there has never been a reliable demonstration of a first order transition when the lightest neutral Higgs weighs at least $O\left(M_{W}\right)$.

In this article we argue that perturbation theory gives a reliable description of a first order transition if $\alpha / \lambda$ and $\alpha / \xi$ are sufficiently big. We present additional arguments for a first order transition based on RG group methods combined with the $\epsilon$-expansion, as well as computation of $V_{\text {eff }}$ based on $1 / N$ expansion techniques. We stress that the first order behavior is not due to gauge fields. We will later address the implications of our results for baryogenesis in the two Higgs-doublet electroweak model.

We study not the full $4 \mathrm{~d}$ model, but the $3 \mathrm{~d}$ effective field theory describing the high $T$ limit of the $4 \mathrm{~d}$ model. Before proceeding with our analysis we introduce all the tools we will use in the context of a simple $\phi^{4}$ model.

Effective 3d Theory. Let us consider the ungauged scalar theory with $4 \mathrm{~d}$ potential

$$
V(\phi)=\frac{\lambda}{4 !}\left(\phi^{2}-v^{2}\right)^{2} .
$$

The model is, at high $T$, formally equivalent to a euclidean field theory with one compact dimension. For physics at scales less than $O(2 \pi T)$ it is sufficient to study the effective 3d Lagrangian that results from integrating out the compact dimension. The $4 \mathrm{~d}$ fields can be expanded as

$$
\phi(\vec{x}, \tau)=\sum_{n} \phi_{n}(\vec{x}) \psi^{n}(\tau),
$$

where $\tau$ parameterizes the compact dimension and the $\psi^{n}(0)=\psi^{n}\left(T^{-1}\right)$. Thus the $4 \mathrm{~d}$ field yields an infinite tower of $3 \mathrm{~d}$ fields when the compact dimension is integrated out in the action. Furthermore, for what we are interested in, only the zero model $n=0$, for which $\psi^{0}(\tau)=\psi^{0}(0)$ is important in the effective $3 \mathrm{~d}$ model because the others pick up large $O(2 \pi T)$ masses from the $4 \mathrm{~d}$ kinetic term.

The largest effect of integrating the nonzero modes out from the theory is to give an $O\left(\lambda T^{2}\right)$ correction to the mass-squared of the zero mode [16]. Therefore, we study an 
effective $3 \mathrm{~d}$ theory with potential

$$
V(\varphi)=\frac{\tilde{\lambda}}{48}\left(T-4 \tilde{v}^{2}\right) \varphi^{2}+\frac{\tilde{\lambda}}{4 !} \varphi^{4},
$$

where we have defined the $3 \mathrm{~d}$ quantities

$$
\varphi=\phi_{0} / \sqrt{T}, \quad \tilde{\lambda}=\lambda T, \quad \tilde{v}^{2}=v^{2} / T
$$

The effective theory can be studied by computing the $3 \mathrm{~d} V_{\text {eff }}$, or by RG methods. The ungauged model is believed to have a second order transition [22]. This result is not accessible by means of high temperature perturbation theory. To see this we need only compute $V_{\text {eff }}$ to one-loop.

The field dependent mass for the potential (5) is

$$
m^{2}(\varphi, T)=\frac{\tilde{\lambda}}{24}\left(12 \varphi^{2}+T-4 \tilde{v}^{2}\right)
$$

We expect the $3 \mathrm{~d}$ model to give a good description of $V_{\text {eff }}(\varphi, T)$ for $m^{2}<4 \pi^{2} T^{2}$, i.e. for $T^{2}$ near $4 v^{2}$ and $\frac{1}{2} \tilde{\lambda} \varphi^{2}<4 \pi^{2} T^{2}$. 五

The one-loop contribution to the effective potential is then $\left(m^{2} \geq 0\right)$

$$
\frac{1}{2} \operatorname{Tr} \ln \left[-\vec{\partial}^{2}+m^{2}\right]=\frac{\Lambda m^{2}}{4 \pi^{2}}-\frac{|m|^{3}}{12 \pi}+\text { const. }
$$

We have used a sharp momentum cutoff to regulate the integral.

The linearly divergent field dependent term is absorbed by renormalizing the $3 \mathrm{~d}$ theory. The field dependent part of the one-loop effective potential is then

$$
V_{\text {eff }}=\frac{\tilde{\lambda}}{48}\left(T-4 \tilde{v}^{2}\right) \varphi^{2}-\frac{|m|^{3}}{12 \pi}+\frac{\tilde{\lambda}}{4 !} \varphi^{4}
$$

This result reproduces the leading temperature dependent part of the so-called daisy sum in the high temperature field theory. Taken at face value, (9) indicates a first order transition with a discontinuous jump in the minimum $|\langle\phi\rangle| \propto \sqrt{\lambda} T$ at a temperature $T_{1}$ slightly above $T_{2}=2 v$. Since we have already indicated that the phase transition is believed to be second order, eq. (9) must not give a reliable determination of $V_{\text {eff }}$. In fact, it is well

\footnotetext{
${ }^{\ddagger}$ We will freely switch back and forth from $3 \mathrm{~d}$ quantities and $4 \mathrm{~d}$ quantities, dropping the zero mode subscripts.
} 
known $[8,9]$ that higher loop corrections are significant. In $3 \mathrm{~d}$, the scalar coupling $\tilde{\lambda}$ has dimension 1 . The dimensionless perturbation parameter is actually $\sim \tilde{\lambda} / m$, a result that can be checked by examining multiloop graphs [8,9]. At $T=T_{2}, \tilde{\lambda} / m \sim \sqrt{\lambda} T / \phi$, and for the apparent minimum at $|\langle\phi\rangle| \propto \sqrt{\lambda} T$ this expansion parameter is of $O(1)$ and therefore perturbation theory cannot be relied upon to distinguish between 1st and 2nd order behaviour.

In the case of an $O(N)$ symmetric model described by (3), $V_{\text {eff }}$ has been computed using $1 / N$ expansion techniques $[23,24,22,8]$ to next-to-leading order. The results indicate that the phase transition is second order $[24,17]$. This method quickly becomes technically complicated, so that $V_{\text {eff }}$ has not been computed beyond next-to-leading order (known results should not be trusted to give a reliable description of what happens for low values of $N)$.

Fortunately, there have been many studies of scalar models based on the renormalization group method [22]. A trajectory in coupling constant space which leads to an infrared stable fixed point under the RG is identified with 2 nd order behaviour. There are two possibilities to study the RG flow equations for a $3 \mathrm{~d}$ theory. One is to work in 4- $\epsilon$ dimensions to some order in $\epsilon$. Although one is interested in $\epsilon=1$, even lowest order results in $\epsilon$ can give useful insight into the nature of the phase transition. 3 The other is to stay in $3 \mathrm{~d}$ and to work to some order in $1 / N$. As with $1 / N$ computations of $V_{e f f}$, leading order results in this expansion are generally only expected to give a good description of the model when there are many scalars.

For a simple $O(N)$ symmetric scalar theory, RG equations have been studied to a high order in both the $1 / N$ expansion and the $\epsilon$-expansion [22]. Both results indicate a 2nd order transition and, in both cases, leading order results give a faithful indication of this. For example, for $N=1$ and 4 d potential (3), the $O(\epsilon)$ and $O\left(\lambda^{2}\right)$ RG flow equation is $[16,22,26]$

$$
\frac{d \lambda}{d t}=\epsilon \lambda-\frac{3}{16 \pi^{2}} \lambda^{2}
$$

Here, $t$ increases in the infrared. This has an infrared unstable fixed point at $\lambda^{*}=0$ and an infrared stable fixed point at $\lambda^{*}=16 \pi^{2} \epsilon / 3$. In the infrared limit, every positive $\lambda$ is

$\S$ The absence of an infrared stable fixed point at $O(\epsilon)$ although not a proof [25] that the model has first order behaviour, may hint at such behaviour for some range of couplings. 
driven to this point.

For an abelian Higgs model, i.e. a gauged $O(2)$ model, with gauge coupling $g$ and $4 \mathrm{~d}$ potential (3) where $\phi^{2}=\phi \cdot \phi$, the RG equations to $O(\epsilon)$ have no infrared stable fixed point $[15,16]$, suggesting that a first order transition may be possible. In fact, high $T$ perturbation theory can be used to give a reliable description of a 1st order transition for $g^{2} \gg \lambda \gg g^{4}[15,7]$. Perturbation theory is reliable because the perturbative expansion parameters go like some (positive) power of $\lambda / g^{2}$ at both minima at $T=T_{1}[10,12,14]$.

For $M_{\text {Higgs }} \geq M_{W}$ the above analysis breaks down. For the abelian Higgs model, a computation of $V_{\text {eff }}$ to next-to-leading order [17,18] in the $1 / N$ expansion gives a second order transition. Futhermore, calculations of critical exponents during a 2nd order transition to next-to-leading order in $1 / N$ remain physical for values of $N \geq 10[29,15]$ suggesting that the prediction of a second order transition for $M_{W} \leq M_{\text {Higgs }}$ and $N \geq 10$ is reliable. In fact, the phase transition is known to be second order for $N=2, M_{W} \leq M_{\text {Higgs }}$ due to other reasons [30]. This demonstrates that the $\mathrm{RG}$ analysis to $O(\epsilon)$ in $4-\epsilon$ dimensions can fail to indicate 2nd order behaviour.

As an aside, we note that much less is well understood when the gauge group is nonabelian. As with the abelian case, there is no infrared stable fixed point to $O(\epsilon)$ in $4-\epsilon \mathrm{d}$. Assuming that infrared divergences in loops due to the self interactions of the magnetic fields are cutoff by a nonperturbatively generated $T$-dependent mass [27], it is again possible to argue $[10,12,13]$ that for $g^{2} \gg \lambda$ the transition is first order. For $g^{2}<O(\lambda), 1 / N$ studies $[18,31]$ suggest that the transition may be second order for a model with enough scalars. For a model with just a few scalars, recent results [32] due to resuming high $T$ perturbation theory in the minimal electroweak model also suggest 2nd order behaviour for $g^{2}<O(\lambda)$. In addition, it has been argued [33] that the existence of a fixed point at $O(\epsilon)$ in $2+\epsilon \mathrm{d}$ may give a reliable indication of a second order transition at $d=3$ and low $N$.

The Toy Model. We are finally ready to demonstrate that the ungauged model described by (2) has a 1st order phase transition for a range of couplings. To our knowledge, what we present below is new.

To begin, we examine the RG equations for (2) in $4-\epsilon$ dimensions. To $O(\epsilon)$, one has 
$[26]$

$$
\begin{aligned}
& \frac{d \lambda}{d t}=\epsilon \lambda-\frac{1}{12 \pi^{2}}\left\{3 \lambda^{2}+\xi^{2}+\xi \alpha+\alpha^{2}\right\} \\
& \frac{d \xi}{d t}=\epsilon \xi-\frac{1}{12 \pi^{2}}\left\{3 \lambda \xi+\lambda \alpha+\frac{1}{2} \xi^{2}+\frac{1}{2} \alpha^{2}\right\} \\
& \frac{d \alpha}{d t}=\epsilon \alpha-\frac{1}{12 \pi^{2}}\left\{\lambda \alpha+\xi \alpha+\frac{3}{2} \alpha^{2}\right\}
\end{aligned}
$$

This has the following fixed points:

$$
\begin{aligned}
\text { i) } & \alpha^{*}=0, \xi^{*}=0, \lambda^{*}=0 \\
\text { ii) } & \alpha^{*}=0, \xi^{*}=0, \lambda^{*}=4 \pi^{2} \epsilon, \\
\text { iii) } & \alpha^{*}=0, \xi^{*}=\frac{24}{13} \pi^{2} \epsilon, \lambda^{*}=\frac{48}{13} \pi^{2} \epsilon .
\end{aligned}
$$

Linear stability analysis reveals the following facts. The fixed point $i$ ), the Gaussian fixed point, is unstable in all directions. The fixed points ii) and iii) are both unstable in the $\alpha$ direction: in the infrared limit, small (positive) values of $\alpha$ are driven larger. The lack of a stable fixed point suggests that the purely scalar model may undergo a first order transition. Indeed, the fact that the coupling $\alpha$ is responsible for the lack of stable fixed points suggests that any first order behaviour will be due to $\alpha$.

We therefore examine high $T$ perturbation theory for $\alpha \gg \lambda$ and $\alpha \gg \xi$. To simplify the analysis, we consider

$$
\lambda=f^{3}, \quad \xi=f^{3}, \quad \alpha=f^{2},
$$

for small positive $f$. This choice of couplings also ensures [14] that all mass scales of interest are much less than $T$, thus allowing us to work with an effective $3 \mathrm{~d}$ theory. Under such circumstances, we may now argue that perturbation theory gives a reliable description of a first order transition. The argument follows closely the one for the abelian Higgs model.

The effective $3 \mathrm{~d}$ potential that we study is

$$
V_{\text {tree }}\left(\varphi_{1}, \varphi_{2}\right)=\frac{\left(f^{3} T\right)}{4 !}\left(\varphi_{1}^{2}+\varphi_{2}^{2}\right)^{2}+\frac{f^{2} T}{6}\left(\varphi_{1} \cdot \varphi_{2}\right)^{2}+\frac{1}{2}\left(\varphi_{1}^{2}+\varphi_{2}^{2}\right) M^{2},
$$

ฯ Our particular assignments are only for numerical convenience and we will later make clearer for what values of couplings we predict first order behaviour. 
where

$$
\begin{aligned}
M^{2} & =\frac{\alpha+5 \lambda}{36} T^{2}-\frac{\lambda}{6} v^{2}=\frac{f^{2}}{36}(1+O(f))\left(T^{2}-T_{2}^{2}\right), \\
T_{2}^{2} & =6 \lambda v^{2} /(\alpha+5 \lambda)=6 f v^{2}(1-O(f)),
\end{aligned}
$$

and $\varphi_{1}, \varphi_{2}$ are the (properly normalized) zero modes of the $4 \mathrm{~d}$ fields $\phi_{1}, \phi_{2}$.

As with the simple scalar model discussed previously, we expect the effective $3 \mathrm{~d}$ theory to give a reliable description of the phase transition as long as all mass scales are less than $2 \pi T$.

The field dependent mass matrix has the eigenvalues

$$
f^{2} \rho_{+}+O\left(f^{3}\right), \quad f^{2} \rho_{-}+O\left(f^{3}\right), \quad f^{2} \sigma_{+}+O\left(f^{3}\right), \quad f^{2} \sigma_{-}+O\left(f^{3}\right)
$$

where

$$
\begin{aligned}
\rho_{ \pm} & =\frac{1}{36}\left(T^{2}-T_{2}^{2}\right) \pm \frac{1}{3} \phi_{1} \cdot \phi_{2}, \\
\sigma_{ \pm} & =\frac{1}{36}\left(T^{2}-T_{2}^{2}\right)+\frac{1}{6}\left[\phi_{1}^{2}+\phi_{2}^{2} \pm \sqrt{\left(\phi_{1}^{2}+\phi_{2}^{2}\right)^{2}+12\left(\phi_{1} \cdot \phi_{2}\right)^{2}}\right] .
\end{aligned}
$$

The first two eigenvalues each have multiplicity 3 .

The one-loop contribution includes a linearly divergent correction which renormalizes the $3 \mathrm{~d}$ theory. The remaining finite contribution is

$$
V_{1-\text { loop }}=-\frac{f^{3}}{12 \pi}\left(3 \rho_{+}^{\frac{3}{2}}+3 \rho_{-}^{\frac{3}{2}}+\sigma_{+}^{\frac{3}{2}}+\sigma_{-}^{\frac{3}{2}}\right)+O\left(f^{4}\right)
$$

At one-loop, we have $V_{\text {eff }}=V_{\text {tree }}+V_{1-\text { loop }}$. At $T=T_{2}$, the real part of the potential has a minimum at $\left|<\phi_{1}>\right|=\left|<\phi_{2}>\right| \sim \frac{T}{15}$ and $\quad<\phi_{1} \cdot \phi_{2}>=O\left(f^{2}\right)$.

Taken at face value, to lowest nontrivial order in $f, V_{\text {eff }}$ implies that the minimum is away from the origin by the time the mass at the origin vanishes and hence that a first order transition has occurred. The dimensionless loop expansion parameters are proportional to

$$
\frac{\lambda T}{m}, \frac{\xi T}{m} \sim \frac{f^{3} T}{m} \text { and } \frac{\alpha T}{m} \sim \frac{f^{2} T}{m}
$$

Here $m$ is a field dependent mass eigenvalue. At $T=T_{2}$, all mass eigenvalues are zero at the origin so that $V_{\text {eff }}$ is not to be trusted near the origin. To verify that (23) gives a reliable prediction of a first order transition we must first demonstrate: i) there is another 
minimum degenerate with the one at the origin at some $T=T_{1}>T_{2}$, and ii) the loop expansion parameters are small at both minima at $T=T_{1}$.

Since the $\phi_{1} \cdot \phi_{2}$ terms are $O\left(f^{2}\right)$ at the minimum at $T=T_{2}$, they do not affect the lowest order (in $f$ ) minimization conditions for $<\phi_{1}>$ and $<\phi_{2}>$. It turns out that for the purposes of discussing the leading order (in $f$ ) characteristics of the phase transition one is allowed to just ignore these terms. To lowest order in $f$, the $3 \mathrm{~d}$ effective potential we study for $T$ close to $T_{2}$ is therefore

$$
V_{\text {eff }}=\frac{\left(f^{3} T\right)}{4 !}\left(\varphi_{1}^{2}+\varphi_{2}^{2}\right)^{2}+\frac{M^{2}}{2}\left(\varphi_{1}^{2}+\varphi_{2}^{2}\right)-\frac{f^{3}}{12 \pi}\left[\frac{1}{36}\left(T^{2}-T_{2}^{2}\right)+\frac{1}{3}\left(\phi_{1}^{2}+\phi_{2}^{2}\right)\right]^{\frac{3}{2}} .
$$

Although this contains a cubic term only at $T=T_{2}$, it still leads to first order behaviour because for $T$ close enough to $T_{2}$ the last term behaves like a cubic term $\propto\left(\phi_{1}^{2}+\phi_{2}^{2}\right)^{\frac{3}{2}}$. One can verify that $T_{1}=T_{2}+O(f) T_{2}$ and $\left|<\phi_{1}>\right|=\left|<\phi_{2}>\right|=O(T)$ at the second minimum at $T=T_{1}$. At the origin, all mass eigenvalues go as $f^{3} T_{2}^{2}$ and thus the loop expansion parameters go as $f \sqrt{f}$ and $\sqrt{f}$. Away from the origin, in the $\phi_{1} \cdot \phi_{2}=0$ direction, the mass eigenvalues only increase and the expansion parameters decrease. Therefore, for sufficiently small $f$, the perturbative expansion is well controlled at both minima at $T=T_{1}$. Just below $T=T_{1}$ the global minimum is away from the origin; this is the signal of first order behaviour.

We end our analysis of the model (2) by discussing three issues:

1) There are values of $\phi_{1} \cdot \phi_{2}$ for which some of the field dependent mass eigenvalues of (14) vanish and hence perturbation theory cannot be reliably used to compute $V_{\text {eff }}\left(\phi_{1}^{2}, \phi_{2}^{2}, \phi_{1} \cdot \phi_{2}\right)$ for such values. For example at $T=T_{2}$, six of the $O\left(f^{2}\right)$ mass eigenvalues $(17),(18)$ vanish at $\phi_{1} \cdot \phi_{2}=0$ suggesting that perturbation theory is not reliable at the apparent minimum away from the origin. Actually, at $T=T_{2}$ and $\phi_{1} \cdot \phi_{2}=0$ these mass eigenvalues go like $O\left(f^{3} T_{2}^{2}\right)$ so that perturbation theory is reliable at the minimum. However, there are still values $\phi_{1} \cdot \phi_{2}=O\left(f \phi_{1}^{2}, f \phi_{2}^{2}\right)$ for which the mass eigenvalues vanish and perturbation theory must be resummed in order to get a more reliable $V_{\text {eff }}$.

2) So far we have said nothing about the transition for $\lambda \sim \xi \sim \alpha$. In this case, since perturbation theory breaks down, we must resort to other methods. Even though the RG analysis in $4-\epsilon \mathrm{d}$ has no infrared stable fixed point to $O(\epsilon)$ the transition may be second order for these values of the couplings. To learn more we can use $1 / N$ expansion techniques 
$[22,23]$ when $\phi_{1}, \phi_{2}$ are $N$-vectors. We have studied two interesting limits:

$$
\begin{gathered}
\text { i) } \quad \xi=\lambda \text { and }\left(N^{2} \lambda\right),(N \alpha) \text { fixed for increasing } N \\
\text { ii) } \quad \alpha=\xi=\lambda \text { and }(N \lambda) \text { fixed for increasing } N .
\end{gathered}
$$

At large $N$, case (22i) leads to first order behaviour while case (22ii) leads to second order behaviour.

To show this last statement it is convenient $[23,17,18]$ to perform the rescalings $\phi_{1} \rightarrow$ $\sqrt{N} \phi_{1}, \phi_{2} \rightarrow \sqrt{N} \phi_{2}$ and $v \rightarrow \sqrt{N} v$. Then, the 3 d effective theory is described by the tree potentials

$$
\text { i) } \begin{aligned}
V= & {\left[\frac{T^{2}}{72}(\lambda+\alpha)-\frac{\lambda}{12} v^{2}\right]\left(\varphi_{1}^{2}+\varphi_{2}^{2}\right)+\frac{\lambda T}{4 !}\left(\varphi_{1}^{2}+\varphi_{2}^{2}\right)^{2}+\frac{\alpha N T}{6}\left(\varphi_{1} \cdot \varphi_{2}\right)^{2}, } \\
\text { ii) } \quad V= & N\left[\frac{T^{2}}{72} \lambda-\frac{\lambda}{12} v^{2}\right]\left(\varphi_{1}^{2}+\varphi_{2}^{2}\right)+\frac{\lambda N T}{4 !}\left(\varphi_{1}^{2}+\varphi_{2}^{2}\right)^{2}+\frac{\lambda N T}{6}\left(\varphi_{1} \cdot \varphi_{2}\right)^{2} \\
& +O(1)
\end{aligned}
$$

for $\lambda \rightarrow \lambda / N^{2}, \alpha \rightarrow \alpha / N$ and $\lambda \rightarrow \lambda / N$, respectively. The $3 \mathrm{~d}$ kinetic terms for both cases are also proportional to $N$, due to the rescalings of the fields.

To compute $V_{\text {eff }}$ in the $1 / N$ expansion one performs the shift

$$
\varphi_{1} \rightarrow \varphi_{1}+\hat{\varphi}_{1} / \sqrt{N}, \quad \varphi_{2} \rightarrow \varphi_{2}+\hat{\varphi}_{2} / \sqrt{N}
$$

dropping all terms linear in the quantum fields $\hat{\phi}_{1}, \hat{\phi}_{2}$ and computes consistently all oneparticle-irreducible diagrams with zero external quantum fields to some order in $1 / N$. The computations are simplified if dimension two auxiliary fields are first used to eliminate the quartic couplings $[22,23]$.

Since the scaling (22i) only confirms our perturbative analysis we will just state how the analysis proceeds. $V_{\text {eff }}$ at $O(N)$ depends only on the combination $\phi_{1} \cdot \phi_{2}$ both at tree level and after all the $O(N)$ diagrams are included. The real part of $V_{\text {eff }}$ is an increasing function of $\phi_{1} \cdot \phi_{2}$ and therefore in the large $N$ limit the vacuum energy is minimized at $\phi_{1} \cdot \phi_{2}=0$. In this limit, $O(1)$ corrections are easy enough to compute; they include the cubic term $-\frac{T}{12 \pi}\left[\frac{\alpha}{3}\left(\phi_{1}^{2}+\phi_{2}^{2}\right)\right]^{\frac{3}{2}}$ which leads to first order behaviour. Note that unlike the perturbative result $(21)$ the cubic term contains no $T^{2}-T_{2}^{2}$ term. This is because such a term is lower order in $N$. 
To study the scaling (22ii) we add the following terms involving dimension two auxiliary fields, $\chi_{+}$and $\chi_{-}$, to the tree potential (23ii):

$$
-\frac{3 N}{2 \lambda T}\left(\frac{\chi_{+}+\chi_{-}}{2}-\frac{\lambda T}{6}\left(\varphi_{1}^{2}+\varphi_{2}^{2}\right)-m^{2}\right)^{2}-\frac{3 N}{2 \lambda T}\left(\frac{\chi_{+}-\chi_{-}}{2}-\frac{\lambda T}{3} \varphi_{1} \cdot \varphi_{2}\right)^{2}
$$

after which the potential becomes to $O(N)$

$$
V=-\frac{3 N}{4 \lambda T}\left(\chi_{+}^{2}+\chi_{-}^{2}\right)+\frac{N}{2} \varphi_{1} \cdot \varphi_{2}\left(\chi_{+}-\chi_{-}\right)+\frac{3 N}{2}\left(\chi_{+}+\chi_{-}\right)\left(\frac{1}{6}\left(\varphi_{1}^{2}+\varphi_{2}^{2}\right)+\frac{M^{2}}{\lambda T}\right)
$$

where $M^{2}=T^{2} \lambda / 36-\lambda v^{2} / 6$. After the shift (24), the Lagrangian is only quadratic in the quantum scalar fields; these can then be integrated out at one-loop to give the leading order effective potential in terms of $\varphi_{1}, \varphi_{2}$ and $\chi_{ \pm}$. Eliminating the auxiliary fields from this effective potential by their equations of motion gives $V_{\text {eff }}\left(\varphi_{1}, \varphi_{2}\right)$ to $O(N)$.

The eigenvalues of the scalar field mass matrix of (26) are just $\chi_{+}$and $\chi_{-}$. Both eigenvalues have multiplicity $N$. Therefore, the $O(N)$ quantum corrections are

$$
\frac{N}{2} \operatorname{Tr} \ln \left[-\vec{\partial}^{2}+\chi_{+}\right]+\frac{N}{2} \operatorname{Tr} \ln \left[-\vec{\partial}^{2}+\chi_{-}\right]
$$

The field dependent divergent part can be absorbed by renormalization of the tree potential and the remaining field dependent finite part yields $\left(\chi_{ \pm} \geq 0\right)$

$$
V_{e f f}=V-\frac{N}{12 \pi}\left(\chi_{+}^{\frac{3}{2}}+\chi_{-}^{\frac{3}{2}}\right)
$$

Here, $V$ is given by (26). The equations of motion for $\chi_{ \pm}$are then

$$
\chi_{ \pm}=\frac{\lambda}{6}\left(\phi_{1} \pm \phi_{2}\right)^{2}+M^{2}-\frac{\lambda T}{12 \pi} \sqrt{\chi_{ \pm}} .
$$

It is now straightforward to verify several facts. For $M^{2} \geq 0, \chi_{ \pm} \geq 0$ and the minimum of the potential is at $<\phi_{1}>=<\phi_{2}>=0$. For $M^{2}<0, \chi_{ \pm}$are both zero at $\phi_{1} \cdot \phi_{2}=0$ and $\lambda\left(\phi_{1}^{2}+\phi_{2}^{2}\right) / 6+M^{2}=0$. Now since $\frac{d V_{\text {eff }}}{d\left(\phi_{1} \pm \phi_{2}\right)^{2}}=\frac{\partial V_{\text {eff }}}{\partial\left(\phi_{1} \pm \phi_{2}\right)^{2}} \propto \chi_{ \pm}$, the point $\chi_{+}=\chi_{-}=0$ has the interpretation $[22,23]$ as the symmetry breaking minimum for $M^{2}<0$. This point moves continuously away from the origin as $T^{2}$ falls continuously below $6 v^{2}$; this is the signal of a second order transition.

Although we have only demonstrated that (2) has a second order transition for the particular case $\lambda=\xi=\alpha$ and at large $N$, we might expect a similar outcome for $N=4$ 
and $\alpha \leq O(\lambda, \xi)$, i.e. the first order behavior driven by $\alpha$ is wiped out by the quantum fluctuations due to $\lambda, \xi$ for small enough $\alpha$. A completely reliable determination may only be possible on the lattice.

3) Finally, in this section, we determine more precisely the ratio $\alpha / \lambda$ for which perturbation theory breaks down completely and the maximum ratio $\frac{\alpha^{2}}{\lambda}$ for which the effective $3 \mathrm{~d}$ theory can be trusted. We examine the one-loop $3 \mathrm{~d}$ potential for $\phi_{1} \cdot \phi_{2}=0, \lambda=\xi$ and dropping for the time being the $T^{2}-T_{2}^{2}$ part of the "cubic" term:

$$
V_{e f f}=\frac{(\lambda T)}{4 !}\left(\varphi_{1}^{2}+\varphi_{2}^{2}\right)^{2}+\frac{M^{2}}{2}\left(\varphi_{1}^{2}+\varphi_{2}^{2}\right)-\frac{\alpha^{\frac{3}{2}}}{12 \pi}\left[\frac{1}{3}\left(\phi_{1}^{2}+\phi_{2}^{2}\right)\right]^{\frac{3}{2}} .
$$

$M^{2}$ and $T_{2}^{2}$ are given by (15) and (16), respectively. For this potential, $T_{1}^{2}-T_{2}^{2}=$ $\alpha^{3} T_{1}^{2} / 9 \lambda \pi^{2}(\alpha+5 \lambda)$, and $M\left(T_{1}\right)=\sqrt{\alpha^{3} / \lambda} T_{1} / 18 \pi$.

Now note that the high $T$ perturbative expansion parameters for (2) are only proportional to $\lambda T / m, \xi T / m, \alpha T / m$. To get an estimate of the constants of proportionality we examined higher loop corrections in the $3 \mathrm{~d}$ theory which grow with the number of scalars. For $\xi=\lambda$, the large $N$ loop expansion parameters for $N=4$ are $\lambda T /(6 \pi m), \alpha T /(6 \pi m)$, where $m^{2}$ is a field dependent mass eigenvalue. At $T_{1}$ these expansion parameters are $\approx 1$ for $\alpha \approx 5 \lambda$. In addition, at $T=T_{1}$ and for $\alpha \geq 5 \lambda$ the $T^{2}-T_{2}^{2}$ term is less than the $\phi_{1}^{2}+\phi_{2}^{2}$ term in the last term of (30), i.e. (30) gives a good description of the potential.

The condition that all field dependent mass eigenvalues are less than $2 \pi T$ at the minimum at $T=T_{2}$ gives $\alpha^{2}<12 \pi^{2} \lambda$. Thus, we predict first order behaviour for around ${ }^{\|}$

$$
\frac{\alpha^{2}}{12 \pi^{2}}<\lambda<\frac{\alpha}{5}
$$

For $\xi=0$ this last lower bound on $\alpha$ is instead around $\lambda<\alpha / 3$.

The Two Higgs Doublet Model. As we have mentioned, the general two Higgs doublet model has 5 scalar quartic couplings, not three. In addition, it has two scalar vevs $v_{1}, v_{2}$, gauge couplings, and dimension two terms which softly break the discrete symmetry $\phi_{1} \rightarrow-\phi_{1}$. The scalar potential is typically written [20]

$$
\begin{aligned}
V= & \lambda_{1}\left(\Phi_{1}^{\dagger} \Phi_{1}-v_{1}^{2}\right)^{2}+\lambda_{2}\left(\Phi_{2}^{\dagger} \Phi_{2}-v_{2}^{2}\right)^{2} \\
& +\lambda_{3}\left[\Phi_{1}^{\dagger} \Phi_{1}+\Phi_{2}^{\dagger} \Phi_{2}-v_{1}^{2}-v_{2}^{2}\right]^{2}+\lambda_{4}\left[\Phi_{1}^{\dagger} \Phi_{1} \Phi_{2}^{\dagger} \Phi_{2}-\Phi_{1}^{\dagger} \Phi_{2} \Phi_{2}^{\dagger} \Phi_{1}\right]
\end{aligned}
$$

\footnotetext{
"We remark that as long as all couplings are perturbative at zero $\mathrm{T}$ these conditions for first order behaviour are not unnatural.
} 


$$
+\lambda_{5}\left[\operatorname{Re}\left(\Phi_{1}^{\dagger} \Phi_{2}\right)-v_{1} v_{2} \cos \zeta\right]^{2}+\lambda_{6}\left[\operatorname{Im}\left(\Phi_{1}^{\dagger} \Phi_{2}\right)-v_{1} v_{2} \sin \zeta\right]^{2}
$$

where the $\lambda_{i}, \zeta$ are real parameters and $\Phi_{1}, \Phi_{2}$ are complex Higgs doublets. We have the following identifications (when $\lambda_{1}=\lambda_{2}, \lambda_{4}=\lambda_{6}=0$ )

$$
\lambda_{1}+\lambda_{3}=\lambda / 6, \quad \lambda_{3}=\xi / 6, \quad \lambda_{5}=2 \alpha / 3
$$

between (2) and (32) with the normalization $\Phi_{1}^{\dagger} \Phi_{1}=\phi_{1}^{2} / 2, \Phi_{2}^{\dagger} \Phi_{2}=\phi_{2}^{2} / 2$.

It is reasonable to ask what can be learnt about the the transition in the two doublet model from the analysis of our toy model. One can convince oneself that (32) will display first order behaviour with the following assumptions: i) $v_{1}=v_{2}$, ii) $\lambda_{5}$ is big enough compared to the other quartic scalar couplings and gauge couplings, and iii) as with the one doublet model, there is a nonperturbatively generated mass for the nonabelian magnetic gauge fields [27]. Conditions i) and ii) ensure that the mechanism for first order behaviour (within perturbation theory) is the same as for our toy model and condition iii) is necessary to ensure that the loop expansion parameter associated with the nonabelian gauge fields is small at small scalar field values at $T=T_{1}$. Although our toy model $(2)$ has $\phi_{1} \cdot \phi_{2}=0$ at tree level, the fact that the $O(\epsilon)$ RG results are independent of terms quadratic in the fields in the tree potential strongly suggests (but does not prove) that we need not require $\cos \zeta$ to be small for (32) to exhibit first order behaviour $(\cos \zeta \neq 1$ generically implies CP violation in the Higgs sector). The perturbative analysis for $\cos \zeta \approx 1$ is more subtle than for our toy model.

However, note that for $\sin \zeta \approx 0, \lambda_{6}\left(\operatorname{Im} \Phi_{1}^{\dagger} \Phi_{2}\right)^{2}=\frac{1}{4} \lambda_{6}\left(\phi_{1} T \phi_{2}\right)^{2}$, with nonzero entries $T_{1,2}=T_{3,4}=-T_{2,1}=-T_{4,3}=1$. We have checked that this term can play the same role as the last term in (2). Thus, we predict first order behaviour when $\sin \zeta \approx 0$ and $\lambda_{6}$ (which determines the pseudoscalar mass $M_{A}$ ) is sufficiently larger than the other couplings, even when the mass of the lightest neutral Higgs $M_{h}$ is near or above $M_{W}$. The precise determination of the parameter space for which first order behaviour is possible will require a more careful analysis, however the ratio $M_{A} / M_{h}$ could be as low as 3 .

Acknowledgements. We are grateful to Rob Leigh and especially Raman Sundrum for interesting and useful discussions.

Note Added. We have just received an interesting article on the electroweak phase transition with a singlet: J.R. Espinosa and M. Quirós, IEM-FT-67/93, hep-ph/9301285. 


\section{References}

[1] M. Dine, P. Huet, R. Singleton and L. Susskind, Phys. Lett. B257: 351 (1991).

[2] M. E. Shaposhnikov, Nucl. Phys. B287: 757 (1987);

A. I. Bochkarev and M. E. Shaposhnikov, Mod. Phys. Lett. A6: 417 (1987);

L. McLerran, M. Shaposhnikov, N. Turok and M. Voloshin, Phys. Lett. B256: 451 (1991);

N. Turok and J. Zadrozny, Phys. Rev. Lett. 65: 2331 (1990).

A. Cohen, D. Kaplan and A. Nelson, Phys. Lett. 245B: 561 (1990) and Nucl. Phys. B349: 727 (1991).

[3] M. E. Shaposhnikov, Phys. Lett. B277: 324 (1992) and Erratum, ibid B282: 483 (1992).

[4] M. E. Shaposhnikov, JETP Lett. 44: 465 (1986), Nucl. Phys. B287: 757 (1987) and Nucl. Phys. B299: 797 (1988).

[5] G. Slade, Comm. Math. Phys. 102: 425 (1985) and 104: 573 (1986);

A. Dannenberg, Phys. Lett. B202: 110 (1988).

[6] C. Itzykson and J.-B. Zuber, Quantum Field Theory, McGraw Hill (1980).

[7] D. A. Kirzhnits and A. D. Linde, Phys. Lett. B42: 471 (1972) and Ann. Phys. 101: 195 (1976).

[8] L. Dolan and R. Jackiw, Phys. Rev. D9: 3320 (1974).

[9] S. Weinberg, Phys. Rev. D9: 3357 (1974).

[10] M. Dine, R. G. Leigh, P. Huet, A. Linde and D. Linde, Phys. Rev. D46: 550 (1992) and Phys. Lett. B283: 319 (1992).

[11] G. W. Anderson and L. J. Hall, Phys. Rev. D45: 2685 (1992).

[12] C. G. Boyd, D. E. Brahm and S. D.H. Hsu, CALT-68-1795, HUTP-92-A027, EFI-92-22.

[13] M. E. Carrington, Phys. Rev. D45: 2933 (1992).

[14] P. Arnold, Phys. Rev. D46: 2628 (1992).

[15] B.I Halperin, T.C. Lubensky and S. K. Ma, Phys. Rev. Lett. 32: 292 (1974).

[16] P. Ginsparg, Nucl. Phys. B170: 388 (1980). 
[17] V. Jain, MPI-Ph/92-72 and hep-ph/9209227.

[18] V. Jain and A. Papadopoulos, LBL-33067 and hep-ph/9210278, to appear in Phys. Lett. B.

[19] ALEPH, DELPHI, L3 and OPAL collaborations, as presented by T. Mori, ICHEP, August 8, 1992, Dallas Texas.

[20] J. F. Gunion, H. E. Haber, G. Kane and S. Dawson, The Higgs Hunter's Guide, AddisonWesley (1990).

[21] A. I. Bockharev, S.V. Kuzmin and M.E. Shaposhnikov, Phys. Lett. 244B: 275 (1990);

N. Turok and J. Zadrozny, Nucl. Phys. B369: 729 (1992).

[22] J. Zinn-Justin, Quantum Field Theory and Critical Phenomena, Oxford University Press (1989) and references therein.

[23] S. Coleman, R. Jackiw and H. D. Politzer, Phys. Rev. D10: 2491 (1974).

[24] R. G. Root, Phys. Rev. D10: 3322 (1974).

[25] T. P. Cheng, E. Eichten and C. F. Li, Phys. Rev D9: 2259 (1974).

[26] J.H. Chen, T.C. Lubensky and D.R. Nelson, Phys. Rev. B17: 4274 (1978),

[27] A. D. Linde, Rep. Prog. Phys. 42: 389 (1979);

T. Appelquist and R. D. Pirarski, Phys. Rev. D23: 2305 (1981).

[28] W. Buchmüller, T. Helbig and D. Walliser, DESY 92-151, Nov. 1992.

[29] S-K. Ma, Phys. Rev. D7: 2172 (1973).

[30] C. Dasgupta and B. I. Halperin, Phys. Rev. Lett. 47: 1556 (1981).

[31] S. Hikami, Prog. Theor. Phys. 64: 1425 (1980).

[32] J. R. Espinosa, M. Quirós and F. Zwirner, CERN-TH.6577/92, December (1992).

[33] J. March-Russell, Phys. Lett. B296: 364 (1992). 